\title{
CO-EVOLUTION ANALYSIS ON COAL-POWER INDUSTRIES CLUSTER ECOSYSTEM BASED ON THE LOTKA-VOLTERRA MODEL: A CASE STUDY OF CHINA
}

\author{
Cui Herui, Peng $\mathrm{Xu}^{*}$ and Zhao Yuqi \\ School of Economics and Management \\ North China Electric Power University, 071003, Baoding, Hebei Province, China \\ donaldpengxu@163.com
}

\begin{abstract}
This contradiction caused by differences in coal-electricity industry market forces is "market for coal, plans for electricity". The traditional coal and power enterprises cause serious pollution problems and ecological problems in the production process, restricting the sustainable socio-economic development. The coal-electricity industry cluster ecosystem forms may effectively mediate organizations conflict existing in the development of industrial clusters, improving resource utilization, reducing energy consumption, to achieve harmony with the natural environment. This paper combines with the theory of industrial ecology, with the Lotka-Volterra model, to explore co-evolution mechanism of coal-electricity industry cluster at the micro, meso and macro levels. In the end, this paper adapted the "Henon mapping" method to describe the chaos phenomenon existed in the development. This paper put forward specific development model for industrial clusters in lower, steady and advanced stages. The co-evolution coal-electricity industry cluster ecosystem is a good solution to numerous conflicts.
\end{abstract}

Key words- Coal-electricity industry cluster, Ecosystem, Lotka-Volterra model, co-evolution analysis

\section{INTRODUCTION}

Coal and power resources are the most important part in China's energy system, and they are also the major support forces for the social and economic construction. Rough resource using patterns in industrial development, natural resource misallocation, waste emissions produced by industrial enterprises and environmental problems are paid more attention recently. The coal industry problems in China have already plagued the people for a long time, seriously restricting the coal industry's development [1].

Firstly, Pollution Problem. It has been acknowledged that coal consumption has a strong correlation with the air quality index. Those years when coal is more produced, the air quality is poorer, and the pollutant emissions from thermal power enterprise account for a large part. The thermal power enterprises release large amounts of pollutants in its production process such as $\mathrm{NO} 2, \mathrm{SO}_{2}$, and $\mathrm{CO} 2$, which seriously 
damaged the human living environment.

Secondly, Organization Problem. Coal industry lies in the upstream of coal-power industries chain and power industry lies in the downstream. Their market forces are significantly different. Generally speaking, coal mines and power plants are often not built in the same area, the differences in regions make coal and power organizations discrete. The "market for coal, plans for electricity" organization structure seriously weakens their production capacity. All these create obstacles in the co-evolution processes for coal and power enterprises. For example, it generates a lot of conflicts between the "Five Power Generation Groups" and "Coal Groups" in the annual coal production and consumption discussion meetings, which directly restricts the development of coal industry. So, how to solve the organization problem of coal enterprise?

Thirdly, Technology Innovation Problem. Coal will produce a lot of pollutants in the combustion process. If the coal and power enterprises reach a technology alliance which encourages the clean production process, the pollutant emissions can be effectively reduced through the technological innovation such as the "Desulphurization and Denitrification Technology" etc. applied in thermal power companies. Furthermore, primary energies like coal are limited. Encouraging the energy technological innovation like the Biomass, Tidal energy, geothermal energy and others can significantly reduce the human's consumer demand for the limited primary energy and achieve sustainable development [2].

Terrain self-purification capacity, if treated as a natural ecosystem, is limited. It will lead to a serious natural crisis, once the produced exhaust gas and pollutants exceed the threshold of terrain self-purification ability. There are methodical interrelated relationships among the various organizations in the ecosystem. And they can achieve sustainable development in ecosystem. At the same time, it has been observed that populations produce wastes, but the wastes can achieve recycling among the various populations in the ecosystem. Modern industries similar to biologic chain are interacting. Industrial ecology theory is developed on the basis.

The "industrial ecosystem" under the artificial system, which can be built by imitating the collaborative development mechanism of populations on the biologic chain, could turn the negative pollutants results to the positive ecology benefit, so that the population's resources can be used efficiently, and get the recovered pollutants and "zero emissions" within the industrial ecosystem, achieving environment-friendly sustainable development in the end [3].

Industry cluster ecosystem is a socio-economic subsystem which based on natural ecosystem. Its main body is enterprise or industry. It contains many characteristics such as open, circulation, regional, level, dynamic, economic and regulatory etc. [4]. It can reduce the pollutant emissions and the production cost through mutual cooperation, organizational and technological innovation within the industry, and achieve the multi-level utilization and recycling of resources. Coal-power industries 
cluster ecosystem uses the combination of basic industrial ecology theory, system engineering and economic laws to operate conventional coal and power industries, achieve a higher socio-economic benefits, improve the resource utilization rate, reduce the destruction to the ecological environment; It can improve production efficiency by selecting the appropriate organization mode, achieve economies of scale and waste recycling and reuse by technology innovation [5].

Some experts and scholars have already done theoretical research in industry ecosystem theory. Fang Wen [6] pointed out related concepts about coal industry ecology and its development mode. Jin Zhao [7] made concrete analysis on the co-evolution mode for internal and external industry and proposed the nested multi-theory analytical framework about co-evolution mode. Caimei Lu [8] proposed the co-evolution analytical framework and system dynamics model, taking hardware clusters in Xiaolan town in Zhongshan City for example. Fang Wang [9] discussed the concept, characteristics and types of ecological chain of information industry clusters ecosystem. Lihong Yu [10] pointed out the vertical arrangement form of coal-power industries chain under the fluctuating demand. Seen from the listed literatures, industry cluster ecosystem provides a systematic analysis method, considering throughout life cycle development process.

This paper constructed coal-power industries clusters ecosystem to solve the contradictions existed in the development process, radically changing the pollution, waste resources, organizational structure contradictory status in the industry ecosystem. The established industry ecosystem stress the environmental factors in the development process, which can achieve perfect integration between coal-power industries clusters and natural ecosystem.

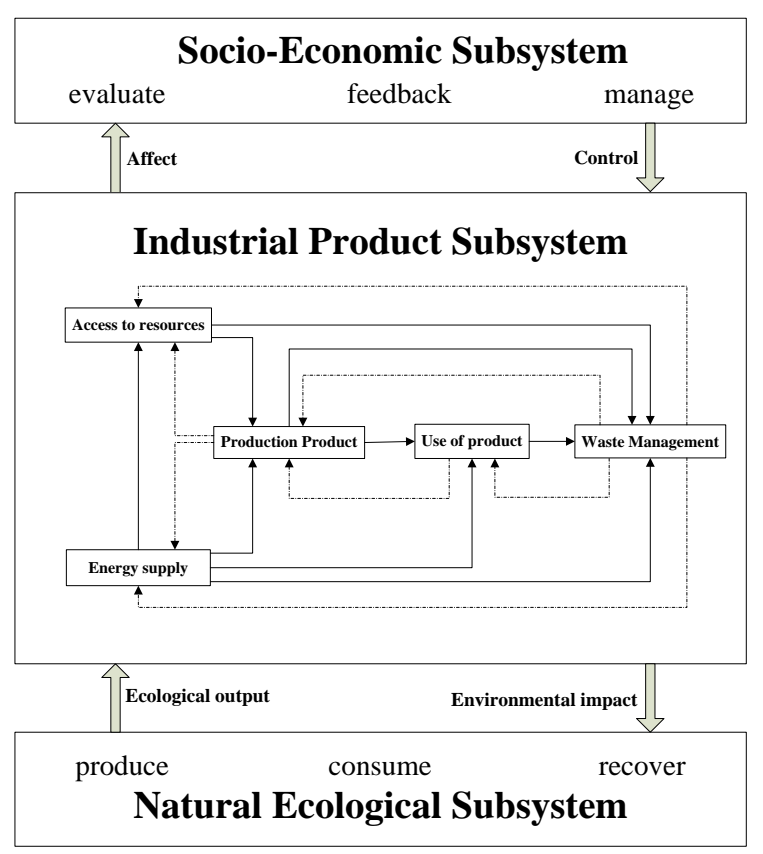

Figure 1. Industrial ecosystem model 


\section{LOTKA-VOLTERRA MODEL}

\subsection{Lotka-Volterra equation}

Lotka-Volterra model was first established by the famous Italian mathematician Volterra in his paper "Fish Changes in Finme Harbor". He studied the number variation and diffusion processes in two competing populations which exist "Predator and Prey Relationships". Then this model is widely applied in the economic field especially between the enterprises [11]. Lotka-Volterra model is used to study the competition and co-evolution development among the industries based on the industry ecosystem theory, which has the similar population relation in the ecosystem [12].

The biological can be divided into several levels depending on the different locations on the food chain in an ecosystem. Lotka-Volterra model was initially used to study the predatory and competition relationship between populations [13].The general form is:

$\frac{d x}{d t}=\gamma_{1} x\left(\frac{k_{1}-\alpha_{12} y-x}{k_{1}}\right)$
$\frac{d y}{d t}=\gamma_{2} y\left(\frac{k_{2}-\alpha_{21} x-y}{k_{2}}\right)$

The $\mathrm{x} \& \mathrm{y}$ indicate the number of two populations in the form; $\gamma_{1}$ and $\gamma_{2}$ respectively represent the natural growth rate; $k_{1}$ and $k_{2}$ represent the maximum environment carrying capacity. The equation can be wrote as the following simplified form:

$\frac{d x}{d t}=x\left[\alpha_{1}+\beta_{1} x+\gamma_{1} y\right]$
$\frac{d y}{d t}=y\left[\alpha_{2}+\beta_{2} x+\gamma_{2} y\right]$

In the above equation, $\beta_{1}, \gamma_{2}$ respectively reflect the density action coefficient in the two populations, which called intraspecific interaction coefficients; $\gamma_{1}, \beta_{2}$ respectively reflect the effect factors between the two interrelated populations, which called interspecific interaction factors[14]; $\alpha_{1}, \alpha_{2}$ represent the natural growth rate of the two populations.

\subsection{Lotka-Volterra equation's balance point and stability}

Make the right side of equation(2) are zero, we get:

$x\left[\alpha_{1}+\beta_{1} x+\gamma_{1} y\right]=0$
$y\left[\alpha_{2}+\beta_{2} x+\gamma_{2} y\right]=0$ abbreviated as $\left\{\begin{array}{l}f(x, y)=0 \\ g(x, y)=0\end{array}\right.$ 
Real roots of equations (3) $x=x^{*}, y=y^{*}$ are called the balance point of equation(3), abbreviated as $M\left(x^{*}, y^{*}\right)$.

For equation (2), we can adopt the approximate linear approach to judge the stability of balance point $M\left(x^{*}, y^{*}\right)$.We can get the approximate linear equation for equation (2) by truncating appropriately the Taylor series expansion at M point.

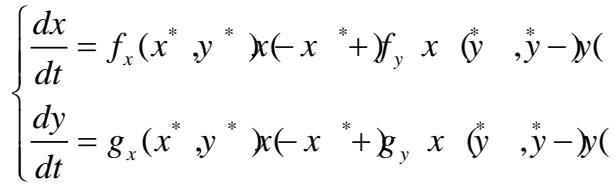

Coefficient matrix $A=\left.\left(\begin{array}{ll}f_{x} & f_{y} \\ g_{x} & g_{y}\end{array}\right)\right|_{M\left(x^{*}, y^{*}\right)} \quad$ Characteristic equation coefficients $P=-\left.\left(f_{x}+g_{y}\right)\right|_{M\left(x^{*}, y^{*}\right)}, \quad q=|A|$; Characteristic equation: $|A-\lambda I|=0$. When $p, q>0$, equilibrium point is stable; when $p, q<0$, equilibrium point is not stable.

Table 1. Species relationship and symbol in Lotka-Volterra Model

\section{No. Interspecific Relationships Relation Features} Symbol Type

1 Mutualism $\quad$ Two organisms living together, beneficial to each other

$$
\gamma_{1}, \quad \beta_{2} \geq 0
$$

2 Predation, prey relationship One party to the other organisms for food

$$
\gamma_{1} \leq 0, \beta_{2} \geq 0
$$

other

$\gamma_{1}, \quad \beta_{2} \leq 0$

The earliest Lotka-Volterra model was just used to study the "Predation - prey relationship" between species; later it is introduced into industrial, economic and other fields to research the competitive relationship between enterprises. Morris [15] et al. proposed a dynamic equation, which described the number changing process of two opponents who have a competitive relationship. Lee [16] then used Lotka-Volterra model to research the price competition between the brokers in Korean and analyzed the impact of two competing markets on the stock market in Korea. Minyan Xu [17] et al. consider the power of industry cluster evolution is competition, cooperation and joint. 
The co-evolution between enterprises in the industry cluster exhibited their own characteristics then she analyzed the co-evolution process between the enterprises in the resource-based industry cluster ecosystem.

Market force is an ability, which manufacturers adopted within a particular industry to interfere the product price in the whole market according to their market position. It's a general phenomenon about market failure. The upstream or downstream companies in a vertical industry chain will influence normal product price with market force by improper access. However, this will lead to inefficient resources allocation in market. The price and quantity of products will not accurately reflect the market supply and demand situation, then the product's price and quantity deviated from equilibrium level, leading to inefficient market ultimately. We may regard the stronger market force part as "Predator role" in the model, and the weaker market force part as "Prey role" in the model. If both market forces are equal, it can be considered as the "mutual competition" relationship.

\section{CONSTITUTION OF COAL-POWER INDUSTRIES CLUSTRIES CLUSTER ECOSYSTEM}

The traditional industrial economics focus on the major enterprise behaviors and market activities acted as "Biological Factor Ingredient", whereas the coal-power industries cluster ecosystem will pay more attention to the non-market activities and non-enterprise behaviors acted as "Non-Biological Factor Ingredient". These factors will influence the surrounding social environment in a broader dimension [18]. So coal-power industries cluster ecosystem should contain the followings.

\section{(1) Technological Innovations}

The traditional coal-power industry chain mode is "Coal - Coal Product Thermal Power - Eliminated Wastes". The traditional linear rough resource consumption methods produced enormous damage to the environment and serious pollution problems. Taking technological transformation and technological innovation such as desulphurization and denitration technological innovation in thermal power plant, clean coal technology, biomass power technology etc. to change the original single linear structure into a network closed energy structure, to recycle and reuse the wasted pollutants, to achieve multi-level use in energy resources, to form a unified, constantly developing, dynamic coal-power industries cluster ecosystem [19]. 


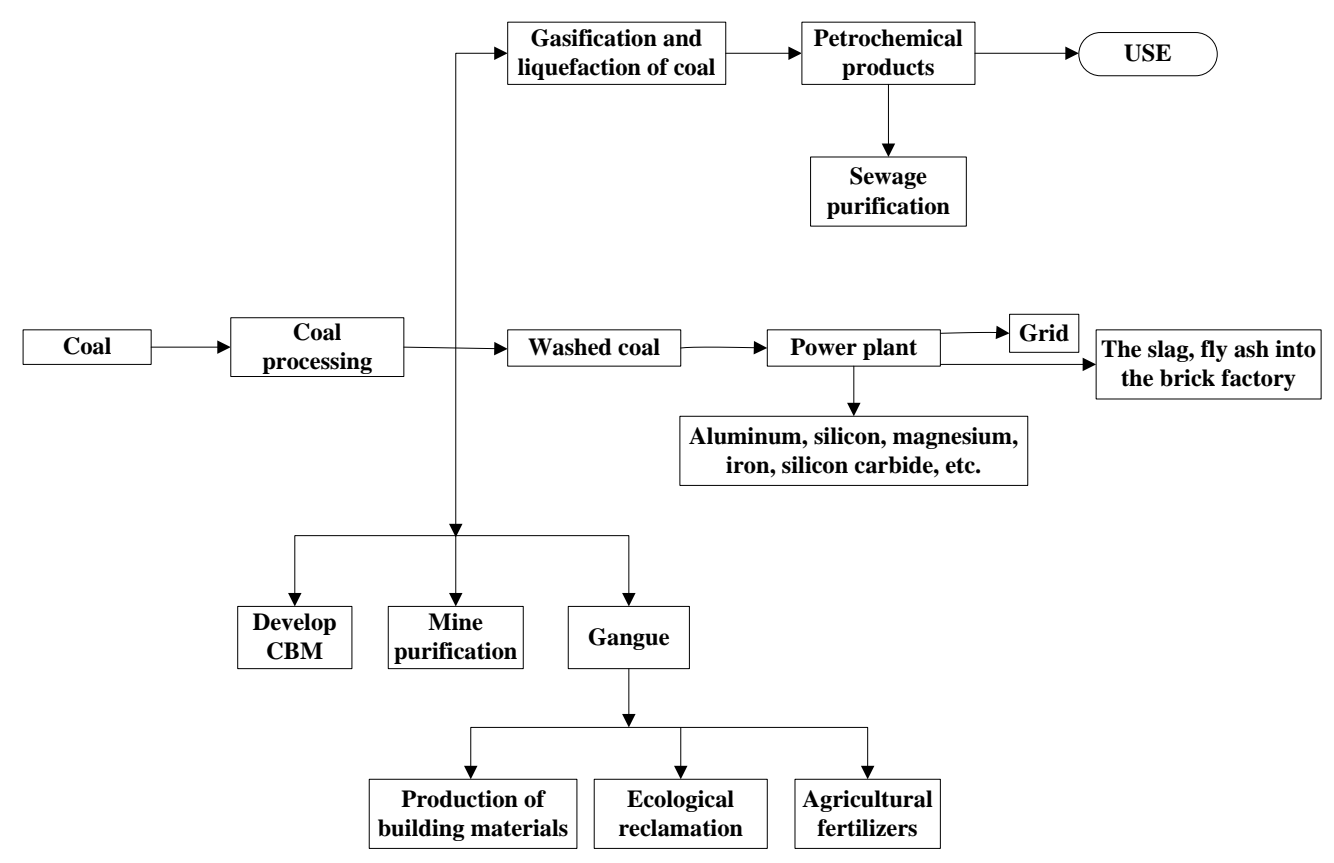

Figure 2. Linear structure of traditional coal-power industry

\section{(2) Motivation and guidance of coal-power industry}

Taking coal linkage, re-nationalization, collaborative management, long-term contracts and other forms, fully extract system elements in the coal-power industries cluster ecosystem through institutional innovation and deepen the cooperation between coal and power companies. Taking institutional innovation in the coal-power industries cluster ecosystem to guide the sustainable development, to stimulate the enterprises' enthusiasm, to enhance the vitality [20]. The government have published "Ecological Consumption", "Coal Enterprise Ecosystem Management" and other regulations. This is the important premise to establish the coal-power industries cluster ecosystem.

\section{(3) Network relationship}

Traditional coal and power industries are isolated different industries. Adapting vertical integration forms such as enterprise mergers, vertical integration, equity control etc. to make the coal and power industries being under the same control, integrate the inter-industry relationships to the internal relations so that transaction costs and production externalities can be effectively reduced and energy efficiency improved [21].

In summary, coal-power industries cluster ecosystem is connected as a whole organic entirety by adapting "biological and non-biological factors" like technical advantages, R\&D, liquidity, technology professionals, government regulation, resource exploitation, regional advantages, cultural organization etc. Adopting mutually beneficial cooperation policy in the industrial chain, making efficiently use of energy resources, providing the necessary power, petrochemical, textile for social and economic development. Coal-power industries cluster ecosystem obtain material energy from nature by means of modern industry, and make production under the government 
management. Coal and power companies in system make coal-power industries cluster and its social, economic, natural environment become a complex ecosystem by technical information, financial support, energy flow, material recycling and other modes [22].

Coal-power industries cluster ecosystem can be subdivided into three levels from lower to higher. They are coal and power "Enterprise Level", coal and power industries "Population Level" and "Community Level". Coal-power industries cluster ecosystem can effectively reduce primary energy consumption such as coal, significantly improve production efficiency of industrial cluster, and realize the nutrient cycling and energy flow in industry cluster ecosystem, achieving the circular economy.

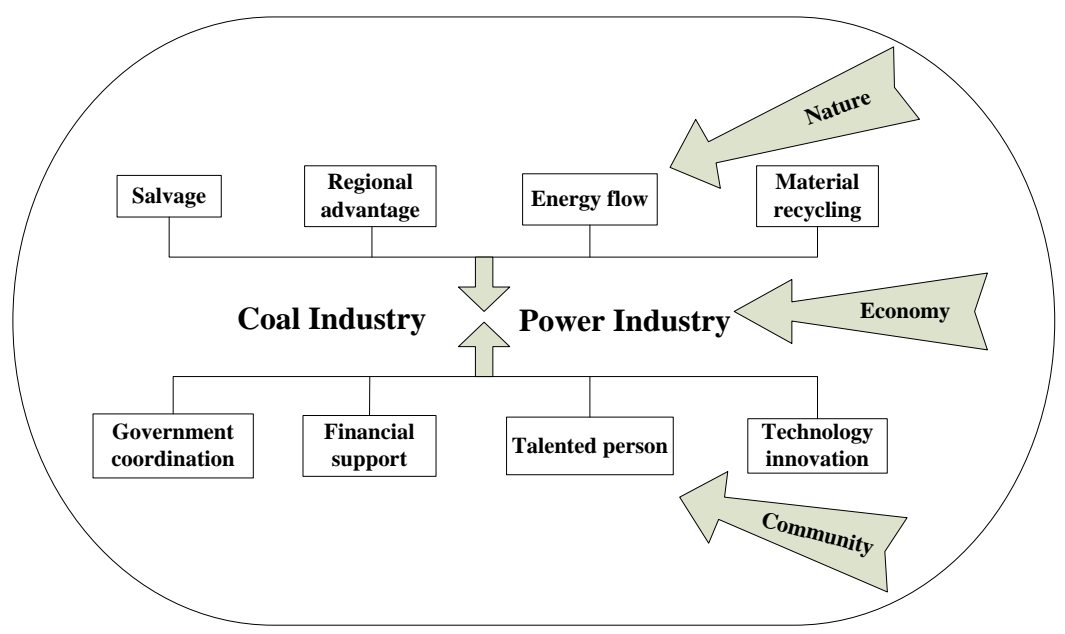

Figure 3. Coal-power industries cluster ecosystem

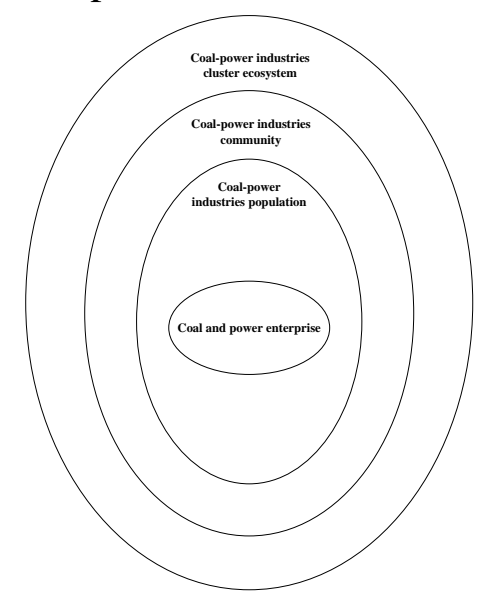

Figure 4. Levels of coal-power industries cluster ecosystem

\section{CO-EVOLUTION MECHANISM OF COAL-POWER INDUSTRIES CLUSTER}

In the previous section, coal-power industries cluster ecosystem is constituted by three levels, "Enterprise level, Population level and Community level". Accordingly, 
coal-power industries cluster ecosystem's co-evolution is launched on three levels. At the enterprise level, coal and power companies fully utilize the resources, and achieving co-evolution through niche overlap and separation; At the population level, coal and power industries achieve co-evolution by competition and cooperation among industries and symbiotic relationship; At the community level, coal and power groups achieve co-evolution through the interaction between communities and surrounding environment [23].

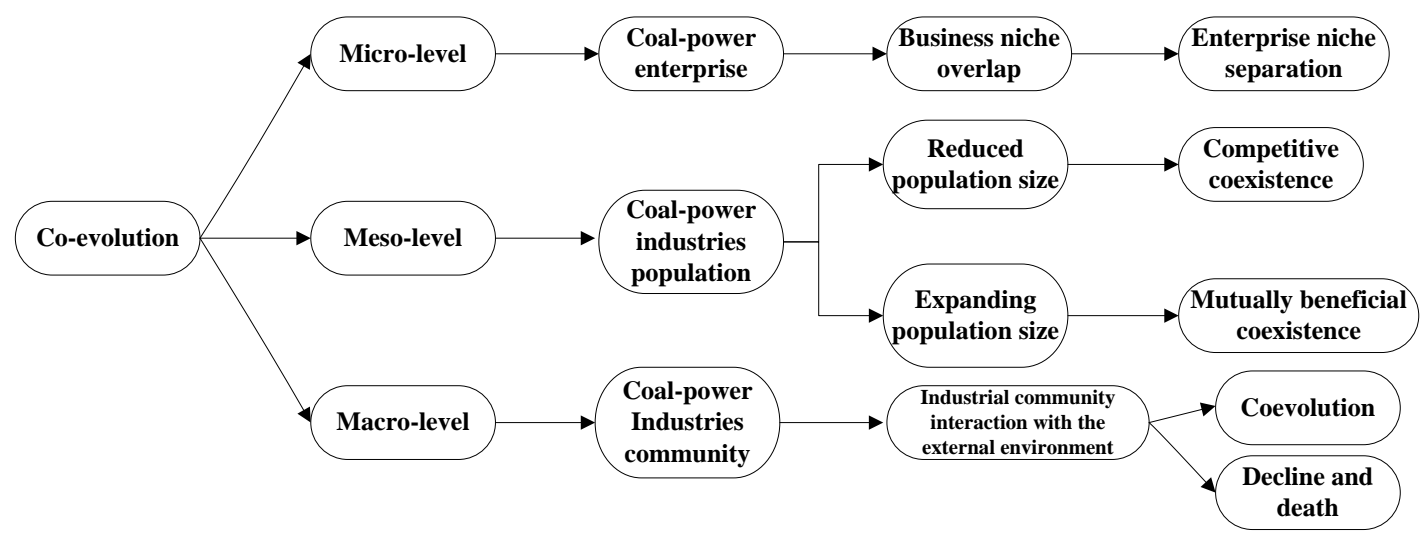

Figure 5. Level and mechanism of coal-power industries cluster ecosystem co-evolution

Coal-power industries cluster ecosystem's co-evolution strategy can be described as: "industry incubation mechanism, industry competition and cooperation mechanism, industry merger and restructuring mechanism" imitating the biological reproduction, competition and genetic recombination behavior in the biological theory [24].

\subsection{Industry incubation mechanism}

Power and coal, which are highly valued by country and government, are important energy power for social and economic construction. Due to the reversed distribution characteristic of energy resource and energy structural contradictions, we must accelerate inter-provincial and inter-regional UHV transmission network line construction, further strengthen the innovation of power generation technology, encourage the construction of wind power, nuclear, biomass, hydropower, improve power generation efficiency, reduce energy consumption and pollution.

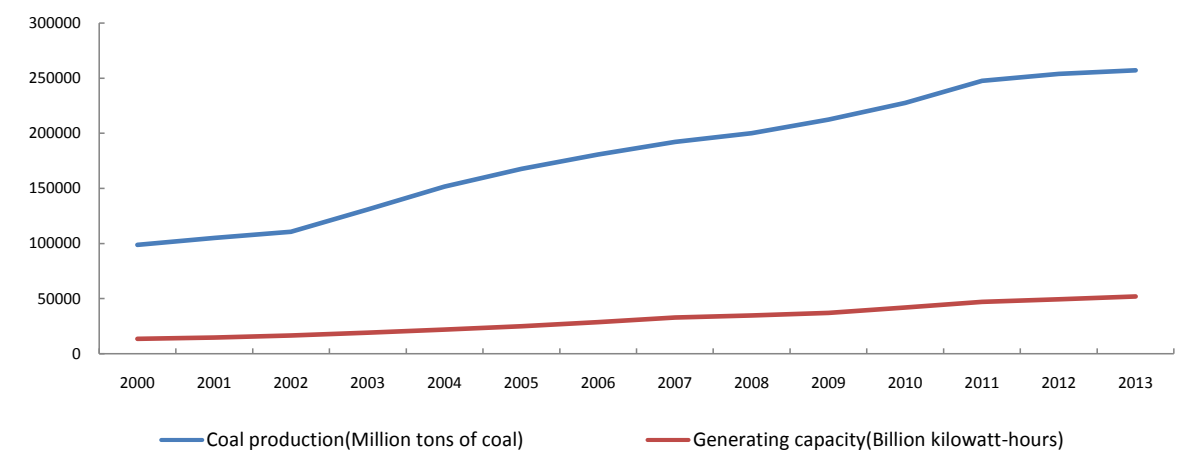


Figure 6. National coal and power production status from 2002 to 2011

Notes : Data from National Bureau of Statistics

\subsection{Competition and cooperation mechanism}

Coal price is now determined by market supply and demand relationship, but the electricity price is still under the government's control. For a long time, coal and power vertical dual pricing, market power differences, the lack of interest linkage mechanism cause lots of conflicts between coal and power companies. Taking coal-electricity linkage policy, establishing pithead power plant change coal energy into electricity energy in order to ease the contradictions, reduce the transportation cost and promote the development of power companies.

\subsection{Merger and restructuring mechanism}

Seen from the vertical relationship, coal industry and power industry separately lie in the upstream and downstream in the same industry chain. The "Thermal Coal" produced by coal enterprises in the upstream is the production factor for power companies in the downstream to generate electricity. Thermal coal has become the link between the coal and power industry chain. This natural inter-industrial relations between coal and power enterprises promote the companies to seek for a breakthrough on the operating mode and put forward vertically integrated business mode like "Coal-Power Joint Venture". Coal and power enterprises hold shares each other, one part acted as the dominant force, develop the unified management, change the traditional industry classification through the forward or backward integration and effectively reduce transaction cost.

\section{CO-EVOLUTION STRATEGIES ANALYSIS USING LOTKA-VOLTERRA MODEL}

The coal-power industries cluster's co-evolution regard the enterprises as evolution actors. It is also a unified part that have interdependence with relevant management organization, supporting institutions such as National Development and Reform Commission, Energy Board, research centers within a certain geographical range; It will be influenced by complex dynamic environment and organizational forms during the development process with characters of self-adaptation, self-reinforcing, feedback regulation; According to the evolution range, it can be divided into micro-evolution, meso-evolution and macro-evolution. The changing numbers about industrial clusters are the surface features, and it may go through the "Generation Development - Maturity - Decline or Evolution" process, pushing each other to achieve the co-evolution. 


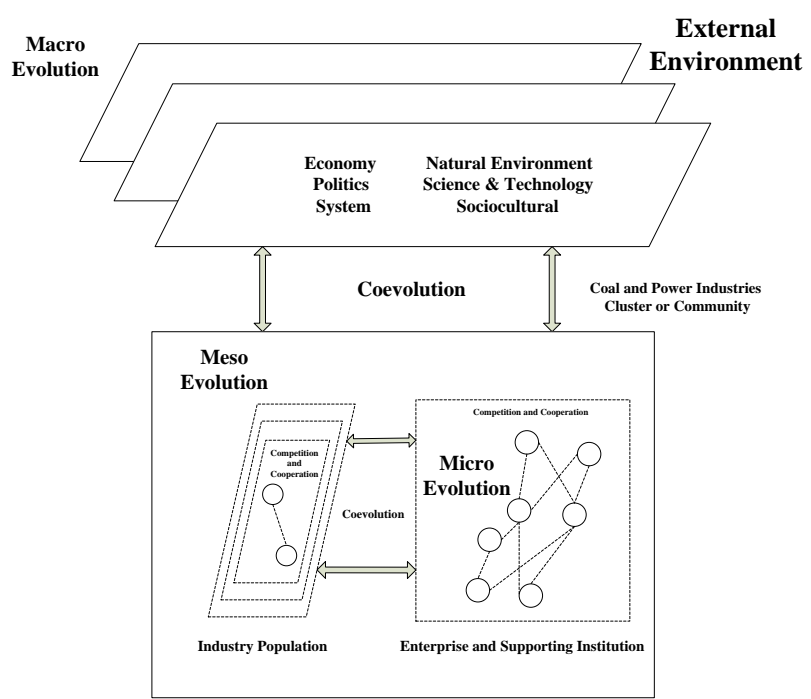

Figure 7. Co-evolution theoretical model

\subsection{Analysis on Lotka-Volterra model}

Establishing coal-power industries cluster ecosystem, applying Lotka-Volterra model from the bionics perspective to mathematically analyze and simulate the future development path of coal and power industries. Lotka-Volterra model:

$$
\begin{aligned}
& \frac{d x}{d t}=x\left[\alpha_{1}+\beta_{1} x+\lambda_{1} y\right] \\
& \frac{d y}{d t}=y\left[\alpha_{2}+\beta_{2} x+\gamma_{2} y\right]
\end{aligned}
$$

It can be known from differential equation, in order to get the equilibrium point of equation (5), making the right side of equation is zero.

$$
\begin{aligned}
& x\left[\alpha_{1}+\beta_{1} x+\gamma_{1} y\right]=0 \\
& y\left[\alpha_{2}+\beta_{2} x+\gamma_{2} y\right]=0
\end{aligned}
$$

We can get four nodes namely origin, intersection point of line $x=0$ and $L_{y}: \alpha_{2}+\beta_{2} x+\gamma_{2} y=0$ located at $\left(0, \frac{-\alpha_{2}}{\gamma_{2}}\right)$, intersection point of line $y=0$ and $L_{x}: \alpha_{1}+\beta_{1} x+\gamma_{1} y=0$ located at $\left(\frac{-\alpha_{1}}{\beta_{1}}, 0\right)$ and intersection point of $L_{y}, L_{x}$ located at $B\left(x^{*}, y^{*}\right)$. Since the different intraspecific and interspecific coefficients $\beta_{1}, \gamma_{1}, \beta_{2}, \gamma_{2}$, equilibrium point will appear the following four cases. 


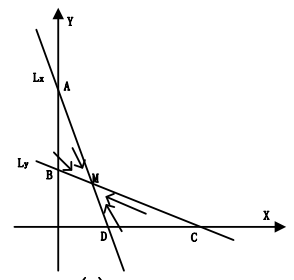

(a)

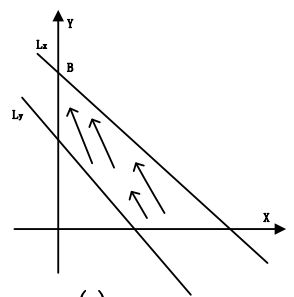

(c)

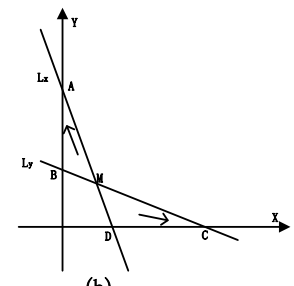

(b)

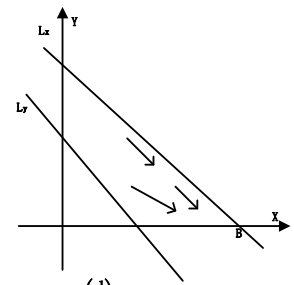

(d)

Figure 8. Rail line graph of Lotka-Volterra model

\subsection{Strategies under steady state}

In subgraph (a) of figure $8,\left(\frac{-\alpha_{1}}{\gamma_{1}}\right)>\left(\frac{-\alpha_{2}}{\gamma_{2}}\right),\left(-\frac{\beta_{2}}{\gamma_{2}}\right)>\left(-\frac{\beta_{1}}{\gamma_{1}}\right) ;$ In the line $L_{x}, L_{y}: \frac{d x}{d t}=0$, $\frac{d y}{d t}=0$; Above the line $L_{x}: \frac{d x}{d t}<0$; Below the line $L_{x}: \frac{d_{x}}{d_{t}}>0$. Individual rail lines have common tangent at the singular point, so zero solution of equation is asymptotically stable nodes. It can be seen from the rail line graph in subgraph $(\mathrm{a}), \lim _{t \rightarrow+\infty} x(t)=x^{*}$, $\lim _{t \rightarrow+\infty} y(t)=y^{*}$ within the first quadrant range and equilibrium point $M\left(x^{*}, y^{*}\right)$ is stable.

Correspondingly, coal and power companies are in a relatively stable period without significant industrial expansion and contraction, and this is also the situation that we are at currently. Coal and power enterprises should take appropriate cooperation and development mechanisms, through establishing institutional measures to guide the development direction of coal-power industries cluster ecosystem. They should focus on vertical integration and specialization, implement the "Coal-Power Linkage", improve labor productivity, reduce the transaction costs, and establish long-term contracts system. Through merger, reorganization, joint cross-regional development, diversified development etc. to significantly enhance the market competitiveness. The evolution belongs to micro-level.

It can be seen from the rail line graph in subgraph (a), you can close the equilibrium point $\mathrm{M}$ from the upper left "Coal industry development region $\Delta_{A B M}$ ", or from the bottom right "Power industry development region $\Delta_{C D M}$ " in order to achieve equilibrium point $\mathrm{M}$. Therefore, we need to integrate the whole coal and power 
industries chain and promote optimal adjustment of the industries chain in order to achieve a balanced state.

From the perspective of the coal industry, power companies located in the downstream in the industry chain may choose merger with the coal companies, implementing the "Backward Integration Strategy. From the perspective of the power industry, coal companies in the upstream in the industry chain can choose merger with power companies, implementing "Forward Integration Strategy". Through technological innovation, making transformation to production equipment, then waste pollution can be largely reduced. Implementing "Coal-Power Linkage", mergers and holding share each other to achieve the overall system optimization, effectively reduce the transaction cost. This concept has been adopted by many scholars and government departments. This evolution belongs to the meso-level.

\subsection{Strategies under unsteady state}

In subgraph (b) of figure 8, if coal and power enterprises follow along the upper left triangle region $\Delta_{A B M}$, the coal industry "y" will be continuous expansion, power industry " $\mathrm{x}$ " will be shrinking[25]. This is the lower stage of co-evolution. During this stage, it has higher utilization rate for coal energy, but the utilization rate for clean energy is low. In the situation, life quality is usually very low. Serious environmental pollution and carbon emissions will be produced and this will not meet the requirements for the social and economic sustainable development. At this stage, we should focus on enhancing technology innovation, increase the use of clean energy sources.

If coal and power enterprises follow along the bottom right triangle region $\Delta_{C D M}$, the coal industry "y" will be shrinking, power industry "x" will be continuous expansion. This is the advanced stage of co-evolution. During this stage, power and other clean energies have become pillar strength in socio-economic development. Coal and other resources consumed by power generation are significantly reduced, and the proportion of biomass power, tidal power, wind power, hydroelectricity have been significantly enhanced. The power industry's dependence on the coal industry is reduced. The carbon emissions have been also reduced, thus coal-power industries cluster ecosystem has achieved the overall optimization, and the adaptability between industrial production and environment is highly suited. Coal and power industries, nature system and social system achieve the harmony evolution. These show that the evolution is macro-level. Similar analysis can be operated to subgraph (c) and (d) in Fig. 8.

\section{CONCLUSIONS}

Industry cluster ecosystem theory study regional industrial system through learning from the co-evolution mode between biological populations in the ecosystem. 
Discussing the relationships between the social \& economic system and natural environment system, to analyze the specific co-evolution mode from the macro view. The main conclusions are follows.

(1) Coal-power industries cluster ecosystem also contain three parts "Nature, Society and Economy". It is organically combined with biological and non-biological system factors such as geographical advantages, financial support, talented person, government coordination, material recycling, energy flow, technological innovation etc..

(2) By Lotka-Volterra model and differential equations, we can describe the specific co-evolution path for coal-power industries cluster ecosystem. At the equilibrium point $\mathrm{M}$, we make a detailed number and economic analysis. Implementing "Coal-Power Linkage", effectively improving industry efficiency and promoting the stable and sustainable development of industry. In non-stationary areas $\Delta_{A B M}$ and $\Delta_{C D M}$, we can adopt the optimization and adjustment for the industry chain, coal and power joint venture, forward or backward integration strategy to reduce the transaction costs, gradually making transition from non-stationary zone to the balance point.

(3) By imitating incubation, competition \& cooperation, recombinant during the biological evolution process, we describe the co-evolution mechanism in the micro, meso and macro-level, namely, "Industry Incubation Mechanism, Industry Competition and Cooperation Mechanism, Industry Mergers and Restructuring Mechanism".

(4) According to the analysis on the Lotka-Volterra model, we can conclude that we should focus on the trend of co-evolution in the coal-power industries cluster ecosystem, preventing extreme events brought by excessive competition which can be described in subgraph (b) and subgraph (c). Wasting resources and over-exploitation are historical retrogression. Strengthening the use of new energy and recycling the polluted waste, taking the road of sustainable development have important meaning.

(5) The co-evolution has different stages, including the lower stage of high coal consumption rate and advanced stage of higher clean energy output, such as the case in Fig.8 (b). In the lower stage, we should focus on promoting technological innovation and institutional guidance, increasing the investment in power and other new energy industries, improving energy efficiency, reducing primary energy consumption and improving the energy consumption structure. And advanced stage is the ideal state in the coal-power industries cluster ecosystem, in which coal consumption will be significantly reduced, the proportion of tidal energy, geothermal energy and other new energy will be significantly increased. Human activities are in harmony with the natural environment. In the stable period, such as the case in Fig.8 (a), this need to be focused on deepening the reform of the coal and power industries, the vertical integration and specialization, enhancing technology innovation, reducing the pollutants emissions and making efforts to achieve the harmony situation. 


\section{REFERENCES}

1. R. Ramanathan, A multi-factor efficiency perspective to the relationships among world GDP, energy consumption and carbon dioxide emissions, Technological Forecasting and Social Change 73, 483-494, 2006.

2. L. Tienan, Energy development report in China 2011, Beijing, Economic Science Press, 2011.

3. ZX. Zhang, Why did the energy intensity fall in China's industrial sector in the 1990s? The relative importance of structural change and intensity change, Energy Economics 25, 625-38, 2003.

4. B. Clarysse, M. Wright, J. Bruneel, A. Mahajan, Creating value in ecosystems: Crossing the chasm between knowledge and business ecosystems, Research Policy 43, 1164-1176, 2014.

5. A. L. Saxenian, Regional Advantage: Culture and Competition in Silicon Valley and Route 128, Harvard University Press, Cambridge, 1996.

6. W. Fang, Coal Industrial Ecology Research in Guizhou Province, Guizhou College of Finance and Economics, 2010.

7. Z. Jin, Evolution Mechanism of Industrial Cluster Cooperative Ecosystem, Beijing Jiaotong University, 2011.

8. L. Caimei and L. Jiahua, Co-evolution model of industrial clusters and Case Studies Xiaolan hardware cluster as an example, China Soft Science 02, 142-150, 2009.

9. W. Fang, Information industry cluster ecosystem ecological chain: Concepts, characteristics and type, Library and Information Service 04, 49-52, 2010.

10. Y. Lihong, Chinese coal industry chain fluctuations in demand and economic arrangements under Regulation longitudinal study, Fudan University, 2006.

11. W. Lifeng, Liu Sifeng, W. Yinao, Grey Lotka-Volterra model and its application, Technological Forecasting \& Social Change 79, 1720-1730, 2012.

12. S. Y. Chiang, An application of Lotka-Volterra model to Taiwan's transition from $200 \mathrm{~mm}$ to $300 \mathrm{~mm}$ silicon wafers, Technological Forecasting and Social Change 79, 383-392, 2012.

13. T. Modis, US Nobel laureates: logistic growth versus Volterra-Lotka, Technological Forecasting and Social Change 78, 559-564, 2011.

14. Y. Tai, J. Y. Lin, M. S. Chen, M. C. Lin, A grey decision and prediction model for investment in the core competitiveness of product development, Technological Forecasting and Social Change 78,1254-1267, 2011.

15. S. A. Morris and D. Pratt, Analysis of the Lotka-Volterra competition equations as a technological substitution model, Technological Forecasting and Social Change 70, 103-134, 2003.

16. S. J. Lee, D. J. Lee, H. S. Oh, Technological Forecasting at the Korean stock market: A dynamic competition analysis using Lotka-Volterra model, Technological Forecasting and Social Change 72, 1043-1056, 2005. 
17. X. Minyan and Z. Heping, Resource-based industry clusters within enterprise collaborative Evolutionary Game Analysis, Enterprise economy 10, 73-75, 2012.

18. G. M. P. Swann, M. Prevezer, D. Stout, The Dynamics of Industrial Clustering: International Comparisons in Computing and Biotechnology, Oxford University Press 30, 353-354, 1998.

19. J.F Moore, The Death of Competition: Leadership \& Strategy in the Age of Business Ecosystems, Harper Business, New York, 1996.

20. F. T. Rothaermel, D. Deeds, Exploration and exploitation alliances in biotechnology: a system of new product development, Strategic Management Journal 25, 201-221, 2004.

21. B. Van Looy, K. Debackere, P. Andries, Policies to stimulate regional innovation capabilities via university-industry collaboration: an analysis and an assessment, $R \& D$ Management 33, 209-229, 2003.

22. S.A. Zahra and S. Nambisan, Entrepreneurship and strategic thinking in business ecosystems, Business Horizons 55, 219-229, 2012.

23. S. Ghirlanda, M. Enquist, J. Lind, Coevolution of intelligence, behavioral repertoire, and lifespan, Theoretical Population Biology 91,44-46, 2014.

24. H. Cabezas, C. W. Pawlowski, A. L. Mayer et al, Simulated experiments with complex sustainable system: Ecology and technology, Resources, Conservation and Recyling 44, 279-291, 2005.

25. M.T. Hannan and J. Freeman, Organizational Ecology, Harvard University Press, Cambridge, 1989. 